\title{
Nuevas poblaciones del helecho amenazado Equisetum moorei Newman en la Comunidad Valenciana (España)
}

\author{
Miguel Angel Gómez-Serrano, \\ 1 Departamento de Microbiología y Ecología. Facultad de Ciencias Biológicas. Universidad de Valencia. E-46100 \\ Burjassot, Valencia, España. \\ ORCID: https://orcid.org/0000-0002-3281-6333 \\ 2 VAERSA, Generalitat Valenciana. Avda. Cortes Valencianas, 20. 46015-Valencia
}

\section{Correspondencia}

Miguel Angel Gómez-Serrano

e-mail: miguel.gomez@uv.es

Recibido: 13 agosto 2019

Aceptado: 25 febrero 2020

Publicado on-line: 24 abril 2020

Editado por: A. Enrique Salvo Tierra

\begin{abstract}
Resumen
Se aportan nuevas citas del helecho amenazado Equisetum moorei en la Comunidad Valenciana, donde solo se conocían previamente seis localidades con presencia actual de la especie. Una de las nuevas poblaciones amplía notablemente el área de distribución conocida en esta región, ya que la mayor parte de las localidades conocidas se concentraban en el macizo del Peñagolosa (provincia de Castellón). Se describen las características ecológicas y demográficas de las nuevas localidades en el contexto de las poblaciones previamente conocidas en la región valenciana.
\end{abstract}

Palabras clave: Castellón, conservación, corología, demografía, ecología, flora amenazada.

\section{Abstract}

New populations of the threatened fern Equisetum moorei Newman in the Valencian Community (Spain)

\begin{abstract}
New records of the threatened fern Equisetum moorei in the Valencian Community are reported, where only six locations were previously known. One of the new populations significantly expands the known range in this region, since most of the known populations were concentrated in Peñagolosa mountains (Castellón province). Ecological and demographic characteristics of the new populations are described in the context of previously known locations in the Valencian region.
\end{abstract}

Key words: Castellon, chorology, demography, conservation, ecology, threatened flora
Equisetum moorei Newman es un taxon de origen hibridógeno, supuestamente entre $E$. hyemale L. y E. ramosissimum Desf., descrito en 1854 por $E$. Newman (Newman, 1854), si bien el lectotipo de esta notoespecie no ha sido propuesto hasta 2017 (Ferrer-Gallego et al., 2017) a partir de material depositado en el herbario de National Botanic Gardens (DBN), en Dublin, Irlanda. A pesar de tratarse aparentemente de un elemento de origen hibridógeno, se ha sugerido que sería más adecuado considerar el taxon como una especie independiente, en base a que presenta un elevado nivel de estabilización, una amplia distribución, e incluso se conoce la presencia de híbridos fértiles (Mateo et al., 2011: 181; Fos, 2016). Aparentemente su distribución estaría condicionada por las regiones ocupadas por sus parentales, estando presente desde áreas del Norte de Europa (Polonia, Rusia) hasta regiones del Sur como Italia (Page, 1990)

Entre los caracteres diagnósticos de Equisetum moorei frente al parental $E$. hyemale, con el que ha sido frecuentemente confundido (Laínz, 1971; Redondo \& Horjales, 1984; Jepson et al., 2013), se han propuesto como esenciales algunos microscópicos relativos a la presencia de esporas fértiles en el caso del parental o la existencia de bandas cruzadas de sílice en las crestas de los tallos del híbrido (Hauke, 1962; Tutin, 1964; Bennert et al., 2005; Jepson et al., 2013). La dificultad de apreciar estas diferencias ha comprometido la correcta identificación de ambos taxones, contribuyendo a la confusión en los datos corológicos disponibles (Aguilella et al., 2010). No obstante, existen algunos caracteres macroscópicos diagnósticos que facilitan la determinación. En este sentido, los más fiables parecen ser: (1) la relación de longitud / anchura de las vainas foliares de la parte media de los tallos (Pétrement et al., 2012; Kalinowski et al., 2016), que en E. moorei es de aproximadamente 2 -hasta 2,3- y en $E$. hyemale de 1 -hasta 1,5-, y (2) la anchura de la banda negra basal de estas vainas foliares, que en el caso de $E$. 
moorei es notoriamente más estrecha (FerrerGallego et al., 2017). Una ilustrativa figura de estos caracteres diagnósticos, basada en la comparación de fotos de las vainas foliares del híbrido y los dos parentales, puede ser apreciada en Jepson et al. (2013: 12).

E. hyemale es un taxón considerado actualmente extinto en la Comunidad Valenciana (Mateo et al., 2011: 181). Fue indicado por Pau (1887) en Segorbe y por Vigo (1968) en el macizo del Peñagolosa. Adicionalmente, ha sido indicada en parajes muy próximos a la provincia de Castellón, pertenecientes al municipio de Iglesuela del Cid, Teruel (Pitarch, 2002). En cambio, existen referencias relativamente recientes de la presencia de E. moorei en varias localidades valencianas (Mateo \& Fabregat, 1991; Mateo et al., 2008), por lo que es probable que estas referencias antiguas de $E$. hyemale se correspondan realmente con poblaciones atribuibles al híbrido (Fos, 2016). Esta sospecha afectaría especialmente a la referencia de E. hyemale de Vigo (1968: 37), quien citó la especie en el Masía del Zurdo (Vistabella del Maestrazgo, Castellón), localidad en la que se conoce una población actual de E. moorei (Mateo \& Fabregat, 1991), sugiriendo que la identificación del parental fue errónea. Por su parte, la cita de Segorbe (Pau, 1887) parece corresponder a E. ramosissimum (Herrero-Borgoñón et al., 1997; Aguilella et al., 2010).

E. moorei es un taxon muy raro y amenazado en la Comunidad Valenciana, estando incluido en el Catálogo Valenciano de Especies de Flora Amenazadas en la categoría de En Peligro de Extinción. En el catálogo original de 2009 (Decreto 70/2009) aparece en esta misma categoría de protección como $E$. hyemale, situación que se cambia en la Orden 6/2013 por la que se modifican los listados valencianos de especies protegidas de flora y fauna, pasando a considerarse únicamente como catalogada la especie E. moorei. Este grado de protección de carácter autonómico permite que la especie se beneficie de actuaciones de conservación, seguimiento poblacional y rastreo de nuevas poblaciones (Servicio de Vida Silvestre, 2017). Fruto de estas prospecciones, se han ido localizando núcleos poblacionales en diversos enclaves del macizo del Peñagolosa, que hasta 2017 sumaban un total de 5 localidades (Tabla 1), 4 si se tiene en cuenta que las poblaciones del barranco del Azor y Molino del Caldero se encuentran en el mismo cauce y cuadrícula UTM de $1 \mathrm{~km}$ de lado. Fuera de este ámbito, en el conjunto de la Comunidad Valenciana únicamente se conoce otra población en el norte de la provincia de Valencia, hallada en 2004 en Andilla (Mateo et al., 2008; Tabla 1), y otra en la Rambla de las Truchas en Vilafranca del Cid, Castellón (Mateo et al., 1995a), cuya presencia no ha vuelto a ser confirmada posteriormente.
Durante las prospecciones llevadas a cabo en 2016 y 2018 por la Tinença de Benifassà y el macizo del Peñagolosa, respectivamente, se pudieron localizar dos nuevas poblaciones de la especie en la provincia de Castellón. La presente nota describe las características ecológicas y demográficas de estas nuevas localidades en el contexto de las poblaciones previamente conocidas en la Comunidad Valenciana.

\section{Equisetum moorei Newman}

Castellón: La Pobla de Benifassà: Fredes, la Canaleta de Mauro, Barranc del Salt hacia el Salt de Robert. UTM 31TBF6110, altitud $940 \mathrm{~m}$, en rezumadero carbonatado bajo pinar negral. 5-XI2016, M.A. Gómez-Serrano (VAL 230735). Barranco del Mojón, Vistabella del Maestrat. UTM 30TYK2262, altitud $1265 \mathrm{~m}$, en barranco umbrío sobre sustrato silíceo y bajo pinar albar. 25-X-2018, M.A. Gómez-Serrano (VAL 242051). Ibidem, UTM 30TYK2162 y UTM 30TYK2163, altitud $1225 \mathrm{~m}$, en barranco umbrío sobre sustrato silíceo. 7-IX-2019, M.A. Gómez-Serrano (v.v.).

Teruel: Barranco del Mojón, Puertomingalvo. UTM 30TYK2262, altitud $1270 \mathrm{~m}$, en barranco umbrío sobre sustrato silíceo y bajo pinar albar. 25-X-2018, M.A. Gómez-Serrano (v.v.). Ibidem, UTM 30TYK2162 y UTM 30TYK2163, altitud $1225 \mathrm{~m}$, en barranco umbrío sobre sustrato silíceo. 7-IX-2019, M.A. Gómez-Serrano (v.v.).

La nueva población localizada en Vistabella del Maestrat (Castellón) se extiende a ambos lados del barranco del Mojón, por lo que pertenece a las provincias de Castellón y Teruel, ya que este tramo del barranco sirve de divisoria provincial. Se han cartografiado un total de 7 núcleos poblacionales que ocupan una superficie de $1.067 \mathrm{~m}^{2}$; parte de los recintos se encuentra en la provincia de Teruel. Por superficie ocupada, se trata de la población más importante en la Comunidad Valenciana (Tabla 1). La población se asienta sobre el lecho y márgenes del barranco y sobre una ladera umbría de substrato silíceo, en el que puntualmente aflora agua generando suelos higroturbosos protegidos por un denso dosel de pino albar. Existe una cita previa Mateo et al. (1995b) en el barranco del Monzón, en la cuadrícula 30TYK2163 de la provincia de Teruel, que aparentemente conectaría con los núcleos aquí localizados. Aunque las plantas de esta población presentan una longitud de las vainas foliares más reducida que el resto de las poblaciones actuales del Macizo de Peñagolosa (Figura 1), los caracteres morfológicos siguen aproximándose más a $E$. moorei que a $E$. hyemale. Las plantas se encontraban en estado vegetativo, no localizándose estróbilos. 

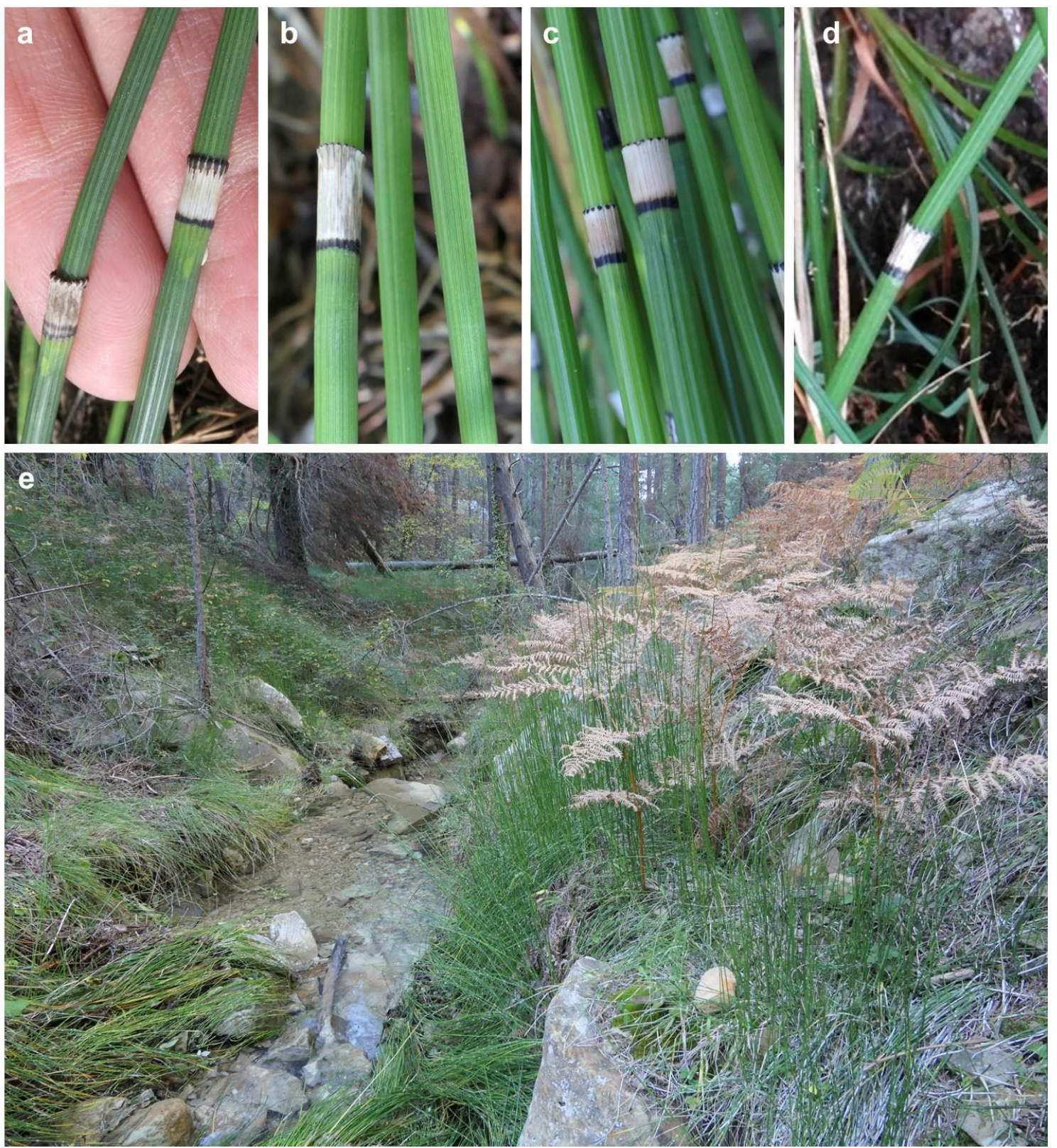

Figura 1. Detalle de las vainas foliares de la parte media de los tallos de plantas de Equisetum moorei procedentes de las poblaciones de (a) Masía del Zurdo, (b) barranco del Azor y (c) barranco del Monzón en Vistabella del Maestrat, y (d) La Pobla de Benifassà. (e) Aspecto general de la población de Equisetum moorei del barranco del Monzón. Todas las imágenes fueron obtenidas en 2018.

Figure 1. Detail of the leaf sheaths in the middle of the shoots of Equisetum moorei plants from the populations of (a) Masía del Zurdo, (b) barranco del Azor and (c) barranco del Monzón in Vistabella del Maestrat, and (d) La Pobla de Benifassà. (e) General appearance of the population of Equisetum moorei from the barranco del Monzón. All images were obtained in 2018.

Por su parte, la nueva población de La Pobla de Benifassà (Castellón) amplía notablemente el área de distribución conocida en la Comunidad Valenciana, ya que aparte de la población de Andilla en el norte de la provincia de Valencia, todas las poblaciones confirmadas en fechas recientes en la provincia de Castellón se concentraban en el macizo del Peñagolosa (Figura 2). Esta nueva población se encuentra en un lugar conocido como la Canaleta de Mauro, junto a una de las sendas valencianas más transitadas, que va desde el caserío de Fredes a los conocidos "Barranc del Salt" y "Salt de Robert".
Dada su ubicación, sorprende que esta población haya permanecido inédita hasta ahora, ya que el paraje es y ha sido frecuentado por numerosos botánicos, al tiempo que puede suponer un factor de riesgo para su conservación. De hecho, en el momento de visitar la población se apreciaron labores de acondicionamiento del punto de agua y la vegetación circundante, incluyendo el clareo de las ramas de un ejemplar de tejo (Taxus baccata L.) que comparte el hábitat con E. moorei. Hay que tener en cuenta que esta población se extiende por una superficie de apenas $50 \mathrm{~m}^{2}$ (Tabla 1), por lo que 
cualquier actuación (especialmente las derivadas de la modificación de las condiciones de hidromorfía del espacio) puede suponer una merma de los efectivos presentes.

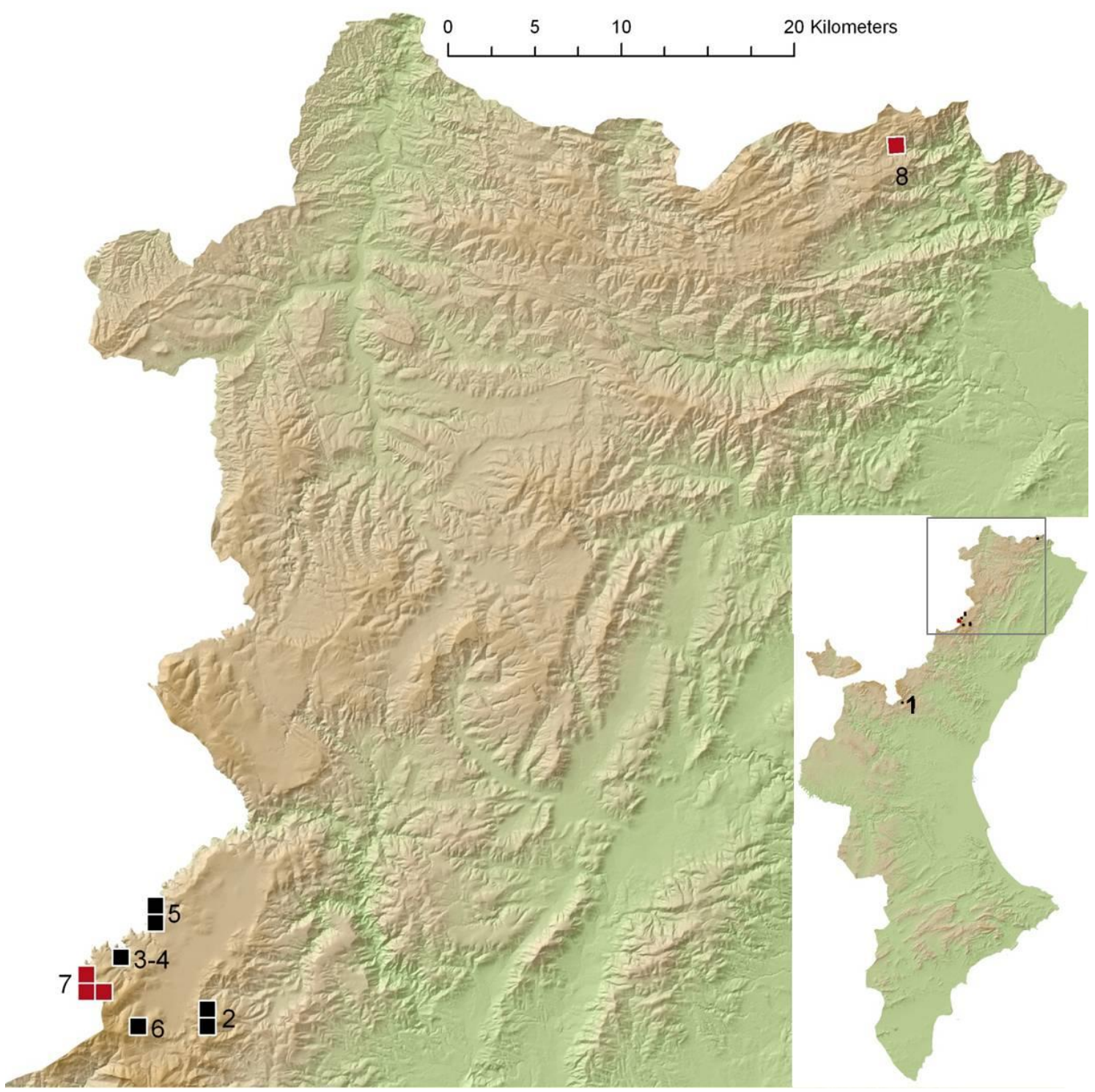

Figura 2. Distribución de las poblaciones actuales de Equisetum moorei en la Comunidad Valenciana. Los cuadros representan cuadrículas UTM de $1 \mathrm{~km}$ de lado (Datum ETRS89). En rojo se muestran las nuevas poblaciones localizadas en este estudio y en negro las conocidas anteriormente. Se muestra ampliado el sector del Norte de la provincia de Castellón donde se encuentran las dos nuevas poblaciones. La numeración de las cuadrículas se corresponde con las siguientes poblaciones: 1 Fuente del Señor, 2 Masía del Zurdo, 3 Barranco del Azor, 4 Molino del Caldero, 5 Masía del Puente, 6 El Manzanar, 7 Barranco del Mojón y 8 Barranc del Salt (Fredes).

Figure 2. Distribution of the current populations of Equisetum moorei in the Valencian Community. The squares represent UTM grids of $1 \mathrm{~km}$ side (Datum ETRS89). The new populations located in this study are shown in red and those previously known in black. The North sector of the province of Castellón where the two new populations are located is expanded. The grid numbers correspond to the following populations: 1 Fuente del Señor, 2 Masía del Zurdo, 3 Barranco del Azor, 4 Molino del Caldero, 5 Masía del Puente, 6 El Manzanar, 7 Barranco del Mojón and 8 Barranc del Salt (Fredes).

La ecología de esta segunda población difiere notablemente del resto de las poblaciones valencianas, ya que las plantas se asientan sobre un rezumadero carbonatado, al contrario del resto de poblaciones, que se encuentran asociadas a barrancos húmedos sobre substratos silíceos. No obstante, este patrón de uso de diferentes tipos de suelos, unos ácidos y otras más carbonatados, es similar al que se observa en otros ámbitos geográficos próximos, como la Serranía de Cuenca, donde existen buenos ejemplos de ambos tipos (Gómez-Serrano \& Mayoral, 2013). 
Al tratarse de una especie catalogada en la Comunidad Valenciana, todas las poblaciones conocidas están incluidas en el Programa de Seguimiento de Flora Amenazada del Servicio de Vida Silvestre, por lo que se posee información detallada y periódica de la demografía y las áreas de ocupación (Fos, 2016). Adicionalmente, todas las poblaciones se encuentran dentro de espacios protegidos. Las localidades del macizo del Peñagolosa y de La Pobla de Benifassà están incluidas dentro de la red Natura 2000, concretamente en los Lugares de Importancia Comunitaria "Penyagolosa" y "Tinença de Benifassà, Turmell i Vallivana", y además, en este último caso, dentro del Parque Natural de la Tinença de Benifassà. Finalmente, la población de Andilla (Valencia) se localiza dentro de la Microrreserva de Flora "Fuente del Señor".

A pesar de esta protección legal de la especie y de los territorios con presencia de la misma, $E$. moorei es una especie relicta cuya conservación plantea importantes retos (lbars et al., 2011). Por un lado, a pesar de conocerse 8 poblaciones diferentes en la Comunidad Valenciana, la superficie ocupada apenas alcanza en conjunto los $2.673 \mathrm{~m}^{2}$ en los últimos censos efectuados en el periodo 2015-2019 (Tabla 1). No obstante, pese a que la serie temporal de datos demográficos es todavía insuficiente, la especie parece mostrar una tendencia poblacional positiva en la región (Servicio de Vida Silvestre, 2017). Por otra parte, aunque se han encontrado híbridos que producen esporas viables, las esporas del género Equisetum se caracterizan por ser viables durante cortos periodos de tiempo, normalmente unos pocos días (Lebkuecher, 1997; lbars et al., 2011). Se han ensayado métodos de crioconservación que han conseguido alargar la viabilidad de las mismas hasta 3 años, e incluso algo más de 6 años introduciéndolas en nitrógeno líquido (Jones \& Hook, 1970; Whittier, 1996; Pence, 2000; Ballesteros et al., 2006; Ibars et al., 2011). Es evidente que, con esta longevidad de las esporas, los bancos de germoplasma no pueden ser una garantía para la conservación de la especie, lo que obliga a dirigir los esfuerzos hacia la conservación in situ, extremando las precauciones frente al deterioro de los hábitats que actualmente ocupa.

Tabla 1. Datos de la localización y demografía de las poblaciones actuales de Equisetum moorei en la Comunidad Valenciana

Table 1. Location and demographic data of the current populations of Equisetum moorei in the Valencian Community

\begin{tabular}{|c|c|c|c|c|c|}
\hline Población & $\begin{array}{l}\text { Municipio } \\
\text { (provincia) }\end{array}$ & UTM 1 km & Referencias $^{1}$ & $\begin{array}{l}\text { Año } \\
\text { último } \\
\text { censo }^{2}\end{array}$ & $\begin{array}{l}\text { Valor último } \\
\text { censo }\left(\mathrm{m}^{2}\right. \\
\text { ocupados) }\end{array}$ \\
\hline Fuente del Señor & Andilla (Valencia) & $30 S X K 8814^{3}$ & Mateo et al., 2008 & 2018 & 310 \\
\hline Masía del Zurdo & $\begin{array}{l}\text { Vistabella del } \\
\text { Maestrat (Castellón) }\end{array}$ & $\begin{array}{l}\text { 30TYK2861 y } \\
\text { 30TYK2860 }\end{array}$ & $\begin{array}{l}\text { Vigo, } 1968 \text { (ut } E . \\
\text { hyemale) } \\
\text { Mateo \& Fabregat, } 1991\end{array}$ & 2016 & 352 \\
\hline $\begin{array}{l}\text { Barranco del } \\
\text { Azor }\end{array}$ & $\begin{array}{l}\text { Vistabella del } \\
\text { Maestrat (Castellón) }\end{array}$ & 30TYKYK2364 & Mateo \& Fabregat, 1991 & 2016 & 188 \\
\hline $\begin{array}{l}\text { Molino del } \\
\text { Caldero }\end{array}$ & $\begin{array}{l}\text { Vistabella del } \\
\text { Maestrat (Castellón) }\end{array}$ & 30TYKYK2364 & Mateo \& Fabregat, 1991 & 2016 & 74 \\
\hline Masía del Puente & $\begin{array}{l}\text { Vistabella del } \\
\text { Maestrat (Castellón) }\end{array}$ & $\begin{array}{l}\text { 30TYK2567 y } \\
\text { 30TYK2566 }\end{array}$ & $\begin{array}{l}\text { Fabregat \& López-Udias, } \\
2008\end{array}$ & 2015 & 264 \\
\hline El Manzanar & $\begin{array}{l}\text { Vistabella del } \\
\text { Maestrat (Castellón) }\end{array}$ & 30TYK2460 & $\begin{array}{l}\text { Navarro et al., } 2013 \text { (en } \\
\text { BDBCV}^{4} \text {; VAL 219528) }\end{array}$ & 2015 & 368 \\
\hline $\begin{array}{l}\text { Barranco del } \\
\text { Monzón }\end{array}$ & $\begin{array}{l}\text { Vistabella del } \\
\text { Maestrat (Castellón), } \\
\text { Puertomingalvo } \\
\text { (Teruel) }\end{array}$ & $\begin{array}{l}\text { 30TYK2262, } \\
\text { 30TYK2162 y } \\
\text { 30TYK2163 }\end{array}$ & $\begin{array}{l}\text { Presente estudio } \\
\text { M.A. Gómez-Serrano } \\
\text { (VAL 242051) }\end{array}$ & $\begin{array}{l}2018- \\
2019\end{array}$ & 1067 \\
\hline $\begin{array}{l}\text { Barranc del Salt } \\
\text { (Fredes) }\end{array}$ & $\begin{array}{l}\text { La Pobla de } \\
\text { Benifassà (Castellón) }\end{array}$ & 31 TBF6110 & $\begin{array}{l}\text { Presente estudio } \\
\text { M.A. Gómez-Serrano } \\
\text { (VAL 230735) }\end{array}$ & 2016 & 50 \\
\hline
\end{tabular}

\footnotetext{
${ }^{1}$ Se indican únicamente las primeras referencias sobre la presencia de la especie en cada localidad.

2 Los valores poblacionales aportados se refieren a los censos oficiales de Flora Catalogada realizados por el personal del Servicio de Vida Silvestre de la Generalitat Valenciana. Se indica el año del último censo y la superficie ocupada por la especie en metros cuadrados.

${ }^{3}$ Citada erróneamente en la publicación original en la cuadrícula 30SXK3814. Se indica la cuadrícula correcta.

${ }^{4}$ BDBCV (Banco de Datos de Biodiversidad de la Comunidad Valenciana; http://www.bdb.gva.es).
}

\section{Agradecimientos}

Pilar Rioja, Leire Gómez y Marina Gómez me acompañaron en buena parte de las prospecciones de campo de Vistabella del Maestrat. Mónica Gil, Pilar Rioja y David González estuvieron presentes en el hallazgo de la población de La Pobla de Benifassà. Simón Fos facilitó la información relativa 
a los censos oficiales de Flora catalogada realizados por el personal del Servicio de Vida Silvestre (Generalitat Valenciana) y revisó un primer borrador del manuscrito. Aruca Sebastián y Carlos Peña colaboraron en la determinación de la población de La Pobla de Benifassà y realizaron el censo de la misma en 2016.

\section{Bibliografía}

Aguilella, A., Fos, S. \& Laguna, E. (Eds.) (2010). Catálogo Valenciano de Especies de Flora Amenazadas. Colección Biodiversidad, 18. Valencia: Conselleria de Medi Ambient, Aigua, Urbanisme i Habitatge, Generalitat Valenciana.

Ballesteros, D., Estrelles, E. \& Ibars, A.M. (2006). Responses of Pteridophyte spores to ultrafreezing temperatures for long-term conservation in Germplasm Banks. Fern Gazette, 17(5), 293-302.

Banco de Datos de Biodiversidad de la Comunidad Valenciana (2019). Sistema de información de Biodiversidad de la Comunidad Valenciana. Conselleria de Agricultura, Desarrollo Rural, Emergencia Climática y Transición Ecológica. Recurso electrónico en http://www.bdb.gva.es/es/. Consulta realizada en 1-08-2019.

Bennert, W., Lubienski, M., Körner, S. \& Steinberg, M. (2005). Triploidy in Equisetum subgenus Hippochaete (Equisetaceae, Pteridophyta). Annals of Botany, 95, 807-815.

Fabregat, C. \& López-Udias, S. (2008). Estudio de plantas amenazadas de la flora local del Macizo de Penyagolosa. Informe inédito. Valencia: Universitat de Valencia-Generalitat Valenciana.

Ferrer-Gallego, P. P., Laguna, E. \& Rossello, J. A. (2017). Typifications of the Linnaean name Equisetum hyemale and $E$. $\times$ moorei (Equisetaceae). Phytotaxa, 305(2), 104-110.

Fos, S. (2016). Equisetum moorei. Ficha del Banco de Datos de Biodiversidad de la Comunidad Valenciana (BDBCV). http://www.bdb.gva.es.

Gómez-Serrano, M.A. \& Mayoral, O. (2013). Flora Amenazada y de interés del Parque Natural de la Serranía de Cuenca. Cuenca: Red de Áreas Protegidas, Junta de Comunidades de Castilla-La Mancha.

Hauke, R.L. (1962). A resumé of the Taxonomic reorganization of Equisetum, subgenus Hippochaete. II-IV. American Fern Journal, 52, 29-35, 57-63, 123-129.

Herrero-Borgoñón, J.J., Martínez-Solís, E., Estrelles, E. \& lbars, A.M. (1997). Avance al atlas pteridológico de la Comunidad Valenciana. Flora Montiberica, 7, 72-86.

Ibars, A.M., Gómez-Serrano, M.A., Mayoral, O. \& Estrelles, E. (2011). Prioridades para la conservación en el ámbito de los helechos en Castilla-La Mancha. In E. Hernández-Bermejo \& J.M. Herranz, Protección de la diversidad vegetal $y$ de los recursos fitogenéticos en Castilla-La Mancha: la perspectiva" in situ" y el compromiso del Jardín Botánico (pp. 189-213). Albacete: Instituto de Estudios Albacetenses Don Juan Manuel.

Jepson, P., Lubienski, M., Llewellyn, P. \& Viane, R. (2013). Hybrids within Equisetum subgenus Hippochaete in England and Wales. New Journal of Botany, 3(1), 47-58.

Jones, L.E. \& Hook, P.W. (1970). Growth and development in microculture of gametophytes from stored spores of Equisetum. American Journal of Botany, 54(4), 430-435.

Kalinowski, P., Sliwinska, E. \& Kruk, J. (2016). Equisetum $\times$ moorei Newman (Equisetaceae)-a "new" nothotaxon in the Polish flora. Biodiversity Research and Conservation, 41, 11-18.

Laínz, M. (1971). Aportaciones al conocimiento de la flora gallega, VII. Instituto forestall de Investigaciones y Experiencias, 12, 1-39.

Lebkuecher, J.G. (1997). Desiccation-time limits of photosynthetic recovery in Equisetum hyemale (Equisetaceae) spores. American Journal of Botany, 84(6), 792-797.

Mateo, G. \& Fabregat, C. (1991). Notes florístiques i corológiques, 492-524. Collectanea Botanica, 20, 239-242.

Mateo, G., Fabregat, C., López-Udias, S. \& Mercadal, N. (1995a). Contribuciones a la flora del Sistema Ibérico, VII. Anales de Biología, 20 (Biología Vegetal, 9), 101-110.

Mateo, G., Fabregat, C. \& López-Udias, S. (1995b). Contribuciones a la flora del Sistema Ibérico, XI. Flora Montiberica, 1, 49-52.

Mateo, G., Crespo, M.B. \& Laguna, E. (2011). Flora Valentina, volumen I. Valencia: Fundación de la Comunidad Valenciana para el Medio Ambiente.

Mateo, G., Torres, C. \& Fabado, J. (2008). Adiciones al catálogo de la flora de las comarcas valencianas de Los Serranos y Ademuz, VIII. Toll Negre, 10, 60-63.

Newman, E. (1854). New Irish Equisetum. Phytologist, 5, 17-20.

Pau, C. (1887). Notas botánicas a la flora española. Fascículo $1^{\circ}$. Madrid.

Page, C. (1990). Hybrids in the genus Equisetum in Europe: an updated annotation. In J. Rita (ed.), Taxonomia, Biogeografia y conservacion de Pteridofitos (pp. 151-156). Palma de Mallorca: IME.

Pence, V.C. (2000). Survival of chlorophyllous and nonchlorophyllous fern spores through exposure to liquid nitrogen. American Fern Journal, 90, 119-126.

Pétrement, B., Bizot, A. \& Krippel, Y. (2012). Equisetum × moorei Newman (Equisetaceae, Pteridophyta), taxon nouveau pour le Luxembourg. Bulletin de la Société des naturalistes luxembourgeois, 113, 83-90.

Pitarch, R. (2002) Estudio de la flora y vegetación de las sierras orientales del Sistema lbérico, La Palomita, Las Dehesa, El Rayo y Mayabona (Teruel). Serie Investigación no 38. Zaragoza: Consejo de Protección de la Naturaleza de Aragón. 
Redondo, N. \& Horjales, M. (1984). Equisetum hyemale L. y otras aportaciones sobre flora vascular gallega. Anales del Jardín Botánico de Madrid, 40(2), 379-384.

Servicio de Vida Silvestre (2017). Evolución de las Poblaciones y Análisis de Tendencias de las Especies del Catálogo Valenciano de Especies de Flora Amenazadas. Año 2016. Valencia: Generalitat Valenciana. Informe inédito. http://www.agroambient.gva.es/documents/91061 501/164096126/IT04_2017+Evolucion+y+tenden cias+especies+CVEFA+2016/ce14c85e-6a064521-af85-f9304d1e2697
Tutin, T.G. (1964). Equisetum L. In: T.G. Tutin, N.A. Burges, A.O. Chater, J.R. Edmondson, V.H. Heywood, D.M. Moore, D.H. Valentine, S.M. Walters \& D.A. Webb (Eds.) Flora Europaea, 1 (pp. 7-9). Cambridge: Cambridge University Press.

Whittier, D.P. (1996). Extending the viability of Equisetum hyemale spores. American Fern Journal, 86(4), 114-118. 